\title{
A Higher Cardiothoracic Ratio Is Associated with 2-Year Mortality after Hemodialysis Initiation
}

\author{
Kiyonori Ito ${ }^{a}$ Susumu Ookawara ${ }^{a}$ Yuichiro Ueda ${ }^{a}$ Haruhisa Miyazawa ${ }^{a}$ \\ Hodaka Yamada $^{b}$ Sawako Goto ${ }^{c}$ Hiroki Ishii $^{a}$ Mitsutoshi Shindo $^{a}$ \\ Taisuke Kitano $^{a}$ Keiji Hirai ${ }^{a}$ Masashi Yoshida ${ }^{b}$ Yoshio Kakua \\ Taro Hoshino $^{a}$ Aoi Nabata ${ }^{a}$ Honami Moria Izumi Yoshida ${ }^{e}$ \\ Masafumi Kakei $^{\text {b }}$ Yoshiyuki Morishita $^{\text {a }}$ Kaoru Tabeia, ${ }^{a}$ \\ Divisions of a Nephrology and ${ }^{b}$ Endocrinology and Metabolism, First Department of \\ Integrated Medicine, Saitama Medical Center, Jichi Medical University, Saitama, 'Division \\ of Clinical Nephrology and Rheumatology, Niigata University Medical and Dental Hospital, \\ and ${ }^{\mathrm{d}}$ Minami-Uonuma City Hospital, Niigata, and e Usui Hospital, Annaka, Japan
}

\section{Key Words}

Cardiothoracic ratio · Diabetes mellitus · Hemodialysis · Ischemic heart disease · Mortality

\section{Abstract}

A high cardiothoracic ratio (CTR) is indicative of a cardiac disorder. However, few reports have revealed an association between the CTR and mortality in patients starting hemodialysis (HD). Methods: Patients with HD initiation ( $n=387$; mean age, $66.7 \pm 12.7$ years) were divided into the following three groups according to their CTR at HD initiation: CTR $<50 \%, 50 \% \leq$ CTR $<$ $55 \%$, and CTR $\geq 55 \%$. Kaplan-Meier analysis was performed to compare 2-year all-cause mortality among these groups. Furthermore, we investigated the factors affecting their 2-year mortality using a Cox proportional hazard regression analysis. Results: Sixty-five patients (17\%) died within 2 years after HD initiation. Kaplan-Meier analysis showed that patients with CTR $\geq 55 \%$ had a higher mortality rate than those in the other groups. Cox proportional hazard regression analysis was performed using parameters with $p$ values $<0.1$ among these three groups [sex, age, presence or absence of ischemic heart disease, hemoglobin levels, serum albumin levels, CTR, body mass index (BMI)] and confounding factors [presence or absence of diabetes mellitus, and estimated glomerular filtration rate (eGFR)]. Age, eGFR, BMI, and CTR $\geq 55 \%$ at HD initiation were identified as factors influencing 2-year mortality. Conclusion: CTR $>55 \%$ is one of the most important independent factors to affect 2-year all-cause mortality. Thus, confirming the cardiac condition of patients at HD initiation with a CTR $>55 \%$ may improve their survival. 
Ito et al.: A Higher Cardiothoracic Ratio Is Associated with 2-Year Mortality after Hemodialysis Initiation

\section{Introduction}

Patients with end-stage renal disease (ESRD) have high rates of coronary artery disease before they initiate hemodialysis (HD) [1]. In addition, in an annual survey of dialysis facilities conducted by the Japanese Society for Dialysis Therapy, heart failure and myocardial infarction were the causes of mortality in approximately one third of all patients undergoing dialysis [2]. Furthermore, cardiac disease is related to intradialytic hypotension [3], and it has been strongly associated with mortality. Therefore, in patients with ESRD, it would be recommended to check for cardiac disease at appropriate times.

The cardiothoracic ratio (CTR) is a clinical parameter used by almost all dialysis facilities in Japan for evaluating body fluid control in patients undergoing HD. The CTR is generally considered normal when it approximates 50\%, and Danzer [4] first described CTR $>50 \%$ as indicative of cardiomegaly. In general, a high CTR signifies that the patient could have some type of cardiac disorder, such as heart failure, left ventricular hypertrophy, or a medical history of ischemic heart disease (IHD). CTR is also an indicator of inflammation and nutritional status in both diabetic and nondiabetic patients stable on maintenance HD; furthermore, it can be used to predict the 2-year all-cause and infection cause mortality in diabetic HD patients, and the 2-year all-cause mortality in nondiabetic HD patients [5, 6]. The relationship between CTR and 2-year mortality in maintenance HD patients had been clearly investigated according to these previous reports; however, the association between the CTR at HD initiation and mortality has been scarcely reported. Furthermore, the range of CTRs that could be considered permissible has not been clarified, even though CTR $<50 \%$ is considered preferable. Therefore, the purpose of this study was to clarify the relationship between the CTR at HD initiation and 2-year mortality in HD patients observed from HD initiation to the maintenance phase.

\section{Materials and Methods}

\section{Study Patients}

This study analyzed the medical records of patients with ESRD who began HD at the Saitama Medical Center, Jichi Medical University, Japan. The included patients were seen in our hospital in and after July 2005, when electronic medical charting began. Of these patients, we included those who had started HD by December 2012 and who were examined using chest radiography at HD initiation.

The patients starting HD were included ( $\mathrm{n}=387 ; 275$ men and 112 women). Patients younger than 20 years of age were excluded. The causes of chronic renal failure were diabetes mellitus (DM; 187 patients), nephrosclerosis (100 patients), chronic glomerulonephritis (50 patients), or other causes (50 patients). Each patient underwent HD 1-3 times weekly, and the duration of each HD session was 3 or $4 \mathrm{~h}$. Table 1 summarizes the characteristics of all included patients.

This retrospective study was approved by the Institutional Review Board of Jichi Medical University, Saitama Medical Center (RIN 14-23), and conforms to the provisions of the Declaration of Helsinki (as revised in Tokyo in 2004). For access to and use of data in the patients' medical charts, a waiver from the Ethics Committee was obtained for this study. Furthermore, in the analysis of this study, personal names as identification names were replaced by a modifier such as a number, and further information with which a patient could be identified was excluded. 
Ito et al.: A Higher Cardiothoracic Ratio Is Associated with 2-Year Mortality after Hemodialysis Initiation

Table 1. Comparison of baseline characteristics of three groups of HD patients at dialysis initiation according to their CTR

\begin{tabular}{|c|c|c|c|c|}
\hline & All patients & CTR $<50 \%$ & $50 \% \leq \mathrm{CTR}<55 \%$ & $55 \% \leq \mathrm{CTR}$ \\
\hline CTR & $52.8 \pm 6.6$ & $45.6 \pm 2.8$ & $52.2 \pm 1.3^{\mathrm{a}}$ & $59.7 \pm 4.4^{\mathrm{a}, \mathrm{c}}$ \\
\hline Patients, n & 387 & 124 & 123 & 140 \\
\hline $\operatorname{Sex}(M / F), n$ & $275 / 112$ & $109 / 15$ & $85 / 38$ & $81 / 59^{a}$ \\
\hline Age, years & $66.7 \pm 12.7$ & $64.2 \pm 12.1$ & $66.0 \pm 12.1$ & $69.4 \pm 11.4^{\mathrm{a}}$ \\
\hline \multicolumn{5}{|l|}{ Primary disease, n (\%) } \\
\hline DM & $187(48.4)$ & $61(49.2)$ & $67(54.5)$ & $59(42.1)$ \\
\hline Nephrosclerosis & $100(25.8)$ & $27(21.8)$ & $26(21.1)$ & $47(33.6)$ \\
\hline Chronic glomerulonephritis & $50(12.9)$ & $21(16.9)$ & $16(13.0)$ & $13(9.3)$ \\
\hline Others & $50(12.9)$ & $15(12.1)$ & $14(11.4)$ & $21(15.0)$ \\
\hline \multicolumn{5}{|l|}{ Past medical history, n (\%) } \\
\hline IHD & $131(33.9)$ & $27(21.7)$ & $30(24.4)$ & $74(53.2)^{\mathrm{b}, \mathrm{c}}$ \\
\hline Cerebral infarction & $57(14.7)$ & $21(16.9)$ & $16(13.0)$ & $20(14.3)$ \\
\hline Smoking & $220(60.7)$ & $71(62.8)$ & $75(64.6)$ & $74(55.6)$ \\
\hline BMI & $23.6 \pm 4.0$ & $22.9 \pm 3.5$ & $23.9 \pm 4.2$ & $24.0 \pm 4.3$ \\
\hline Systolic BP, mm Hg & $151 \pm 26$ & $151 \pm 23$ & $154 \pm 25$ & $147 \pm 28$ \\
\hline Heart rate/min & $79 \pm 14$ & $78 \pm 13$ & $79 \pm 15$ & $79 \pm 15$ \\
\hline Total protein, g/dl & $6.2 \pm 0.7$ & $6.2 \pm 0.7$ & $6.1 \pm 0.8$ & $6.2 \pm 0.7$ \\
\hline Albumin, g/dl & $3.1 \pm 0.7$ & $3.2 \pm 0.7$ & $3.0 \pm 0.7$ & $3.1 \pm 0.6$ \\
\hline Serum sodium, meq/l & $137 \pm 5$ & $137 \pm 4$ & $137 \pm 4$ & $137 \pm 5$ \\
\hline Serum potassium, meq/l & $4.6 \pm 0.9$ & $4.7 \pm 1.0$ & $4.6 \pm 0.8$ & $4.6 \pm 0.9$ \\
\hline Serum calcium, mg/dl & $8.0 \pm 1.0$ & $8.1 \pm 0.9$ & $7.9 \pm 1.0$ & $7.9 \pm 0.9$ \\
\hline Serum phosphate, $\mathrm{mg} / \mathrm{dl}$ & $6.1 \pm 1.3$ & $6.1 \pm 1.5$ & $6.4 \pm 1.7$ & $5.9 \pm 1.6$ \\
\hline $\mathrm{BUN}, \mathrm{mg} / \mathrm{dl}$ & $86 \pm 27$ & $85 \pm 28$ & $90 \pm 26$ & $84 \pm 29$ \\
\hline $\mathrm{eGFR}, \mathrm{ml} / \mathrm{min} / 1.73 \mathrm{~m}^{2}$ & $5.8 \pm 2.3$ & $5.8 \pm 2.2$ & $5.6 \pm 2.2$ & $6.0 \pm 2.4$ \\
\hline Uric acid, mg/dl & $8.1 \pm 2.2$ & $8.0 \pm 2.0$ & $8.1 \pm 2.3$ & $8.1 \pm 2.2$ \\
\hline Blood glucose, mg/dl & $145 \pm 63$ & $139 \pm 59$ & $148 \pm 62$ & $146 \pm 67$ \\
\hline $\mathrm{HbA1c} \%$ & $5.9 \pm 1.0$ & $5.9 \pm 1.0$ & $5.8 \pm 0.9$ & $5.9 \pm 1.1$ \\
\hline Hemoglobin, g/dl & $8.6 \pm 1.7$ & $9.1 \pm 1.7$ & $8.5 \pm 1.5^{\mathrm{a}}$ & $8.2 \pm 1.6^{b}$ \\
\hline TSAT, $\%$ & $23 \pm 17$ & $25 \pm 17$ & $23 \pm 16$ & $21 \pm 16$ \\
\hline Ferritin, ng/dl & $209 \pm 391$ & $228 \pm 496$ & $224 \pm 449$ & $180 \pm 239$ \\
\hline Total cholesterol, mg/dl & $161 \pm 48$ & $159 \pm 48$ & $165 \pm 47$ & $160 \pm 49$ \\
\hline HDL cholesterol, mg/dl & $40 \pm 14$ & $41 \pm 17$ & $39 \pm 13$ & $40 \pm 12$ \\
\hline C-reactive protein, mg/dl & $2.5 \pm 6.9$ & $2.1 \pm 9.8$ & $2.3 \pm 4.9$ & $2.9 \pm 4.9$ \\
\hline \multicolumn{5}{|c|}{ Medication, $\mathrm{n}(\%)$} \\
\hline Renin-angiotensin system blocker & $264(68.2)$ & $90(72.6)$ & $88(71.5)$ & $86(61.4)$ \\
\hline Calcium channel blocker & $305(78.8)$ & $97(78.2)$ & $101(82.1)$ & $107(76.4)$ \\
\hline Beta-blocker & $183(47.2)$ & $54(43.5)$ & $56(45.5)$ & $73(52.1)$ \\
\hline Vitamin D analog & $224(57.8)$ & $75(60.4)$ & $76(61.7)$ & $73(52.1)$ \\
\hline Statin & $113(29.1)$ & $30(24.1)$ & $42(34.1)$ & $41(29.1)$ \\
\hline Antiplatelet agents & $162(41.8)$ & $43.5(53.4)$ & $44(35.7)$ & $64(45.7)$ \\
\hline Erythropoiesis stimulating agent & $366(94.5)$ & 117 (94.3) & $116(94.3)$ & $133(95.0)$ \\
\hline
\end{tabular}

BUN = Blood urea nitrogen; HbA1c = glycated hemoglobin; TSAT = transferrin saturation; HDL = highdensity lipoprotein.

a Significant compared with the CTR $<50 \%$ group, $\mathrm{p}<0.01$. ${ }^{\text {b }}$ Significant compared with the CTR $<50 \%$ group, $\mathrm{p}<0.001$. $^{\mathrm{c}}$ Significant compared with the $50 \% \leq \mathrm{CTR}<55 \%, \mathrm{p}<0.001$.

\section{Data Collection}

Data in the patients' medical charts, including baseline characteristics, laboratory data, and medications, were reviewed. Blood pressure (BP) and heart rate were measured with patients in the supine position before the first dialysis session. The body mass index (BMI) at the first HD was calculated as weight $(\mathrm{kg})$ divided by the square of height $\left(\mathrm{m}^{2}\right)$. All biochemical 
Ito et al.: A Higher Cardiothoracic Ratio Is Associated with 2-Year Mortality after Hemodialysis Initiation

variables were measured before the first HD. The estimated glomerular filtration rate (eGFR) was calculated using the Japanese equation [7] as follows:

eGFR $\left(\mathrm{ml} / \mathrm{min} / 1.73 \mathrm{~m}^{2}\right)=194 \times \mathrm{Cr}-1.094 \times$ age -0.287 ( $\times 0.739$ for women),

where $\mathrm{Cr}$ represents the serum creatinine level $(\mathrm{mg} / \mathrm{dl})$ and the unit of age is years. The eGFR for each patient of this study is the residual eGFR at the first HD initiation as an evaluation of patients' renal function. The past medical history regarding IHD, cerebrovascular disease, and smoking was confirmed on the basis of medical records rather than on examination in our hospital. Medication use was defined as the use of a medicine by a patient at the time of HD initiation.

We included patients undergoing HD for whom chest radiography was performed in the posterior-anterior view within 2 weeks of HD initiation. In most patients, chest radiography was performed on the day of HD initiation or within 3 days after initiation. Based on the results of chest radiography, the CTR in each patient was measured by two or more physicians.

Additionally, we investigated the 2-year survival after HD initiation of all included patients by checking outpatient medical records in our hospital and confirming the identity of the maintenance HD facility.

\section{Statistical Analysis}

Data are presented as the mean and standard deviation. One-way analysis of variance was used to test for significance among the three CTR groups. Furthermore, the correlation between various clinical parameters and the CTR was evaluated using Pearson's correlation coefficient. Variables that had a significant correlation with CTR in a simple linear regression analysis were included in the multivariable linear regression analysis to clarify factors determining CTR in HD patients at dialysis initiation. A p value $<0.05$ was considered statistically significant. Kaplan-Meier analysis and the log-rank test were used to compare 2-year allcause mortality among these three groups. In this study, factors that tended to be different among these three groups, including the presence or absence of IHD ( $<<0.1)$, and confounding factors from previous reports, including the presence or absence of DM and eGFR [8-11], were applied in a Cox proportional hazard regression analysis, which was performed to evaluate risk factors for all-cause mortality using stepwise analysis. Data were analyzed using EZR (Saitama Medical Center, Jichi Medical University), a basic statistics graphical user interface for $\mathrm{R}$ [12] (EZR is a modified version of the $\mathrm{R}$ commander added to statistical functions that is commonly used in the medical field; http://www.jichi.ac.jp/saitama-sct/ SaitamaHP.files/statmedEN.html).

\section{Results}

The patients' demographic and clinical characteristics are shown in table 1 . The patients' mean age was $66.7 \pm 12.7$ years, and the rates of IHD and DM were 33.9 and $48.8 \%$, respectively. The mean CTR among all patients was $52.8 \pm 6.6 \%$, and the mean CTRs in the CTR $<50 \%$ (group 1), $50 \% \leq \mathrm{CTR}<55 \%$ (group 2), and CTR $\geq 55 \%$ (group 3 ) groups were $45.6 \pm$ $2.8,52.2 \pm 1.3$, and $59.7 \pm 4.4 \%$, respectively. As shown in figure 1, a histogram combining all patients' CTR data revealed that $50 \% \leq$ CTR $<55 \%$, namely group 2 , comprised the largest segment compared to other CTR segments. Furthermore, as shown in figure 2, a histogram assessing the presence or absence of IHD revealed that the CTR in patients with IHD was higher than that in patients without IHD. On the contrary, the CTR in patients with DM was lower than that in patients without DM (fig. 3).

Patients' characteristics and the comparisons of clinical parameters among the three groups are displayed in table 1 . There are significant differences in CTR, sex, age, the presence 
Ito et al.: A Higher Cardiothoracic Ratio Is Associated with 2-Year Mortality after Hemodialysis Initiation

Fig. 1. Histogram of the CTR in all patients at HD initiation.

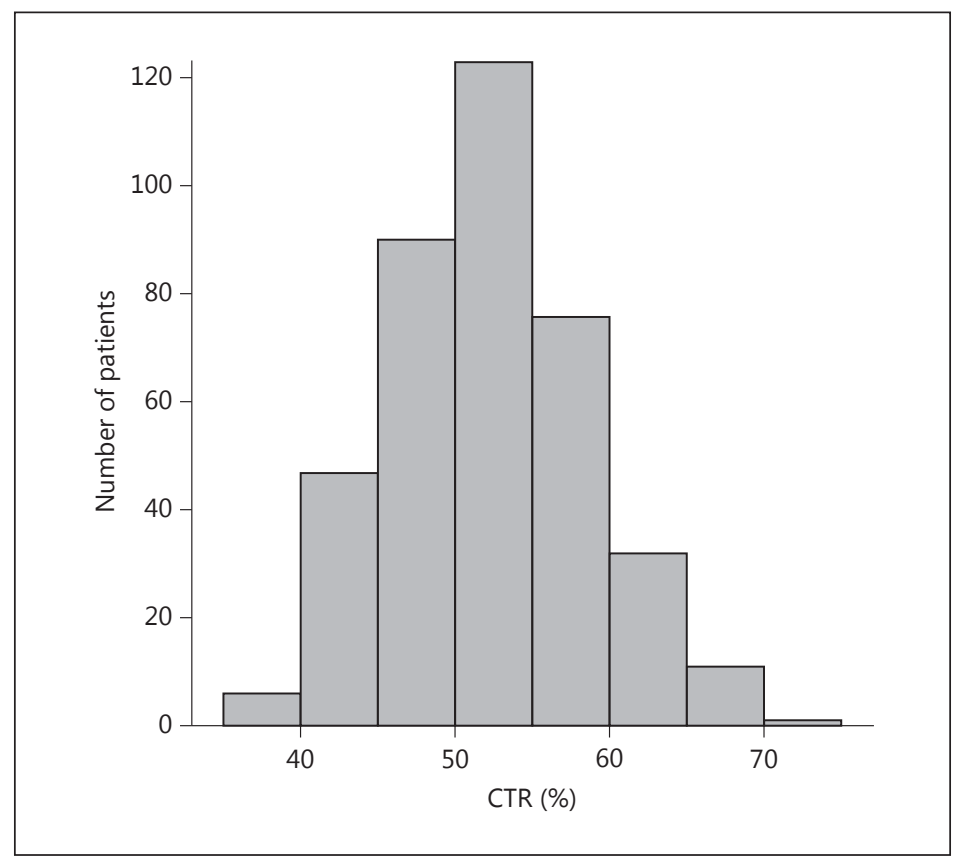

Fig. 2. Histogram of the CTR according to the presence or absence of IHD at HD initiation.

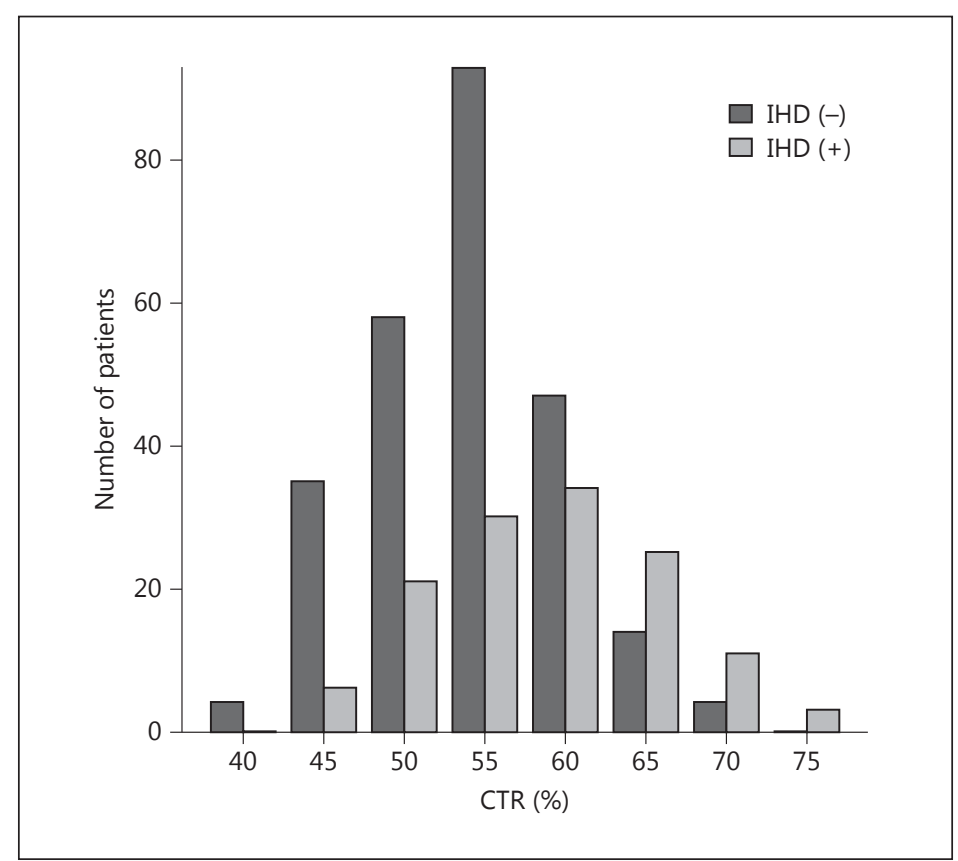

or absence of IHD, and hemoglobin levels; moreover, BMI ( $p=0.073)$ and serum albumin ( $p=0.05$ ) tend to be different among these groups. In addition, as shown in table 2, the CTR was positively correlated with age and BMI, and negatively correlated with hemoglobin levels and serum albumin in a simple linear regression analysis. The multivariable regression analysis including variables that were significantly correlated with CTR in the simple linear regression analysis showed that CTR was independently associated with BMI (standardized coefficient: 0.231$)$, age $(0.214)$, and hemoglobin levels ( -0.197$)$.

Furthermore, we confirmed that 65 patients undergoing HD (17\%) died within 2 years after HD initiation. Kaplan-Meier analysis was performed for the three CTR groups (fig. 4). 
Fig. 3. Histogram of the CTR according to the presence or absence of DM at HD initiation.

Table 2. Determinants of CTR in HD patients at dialysis initiation

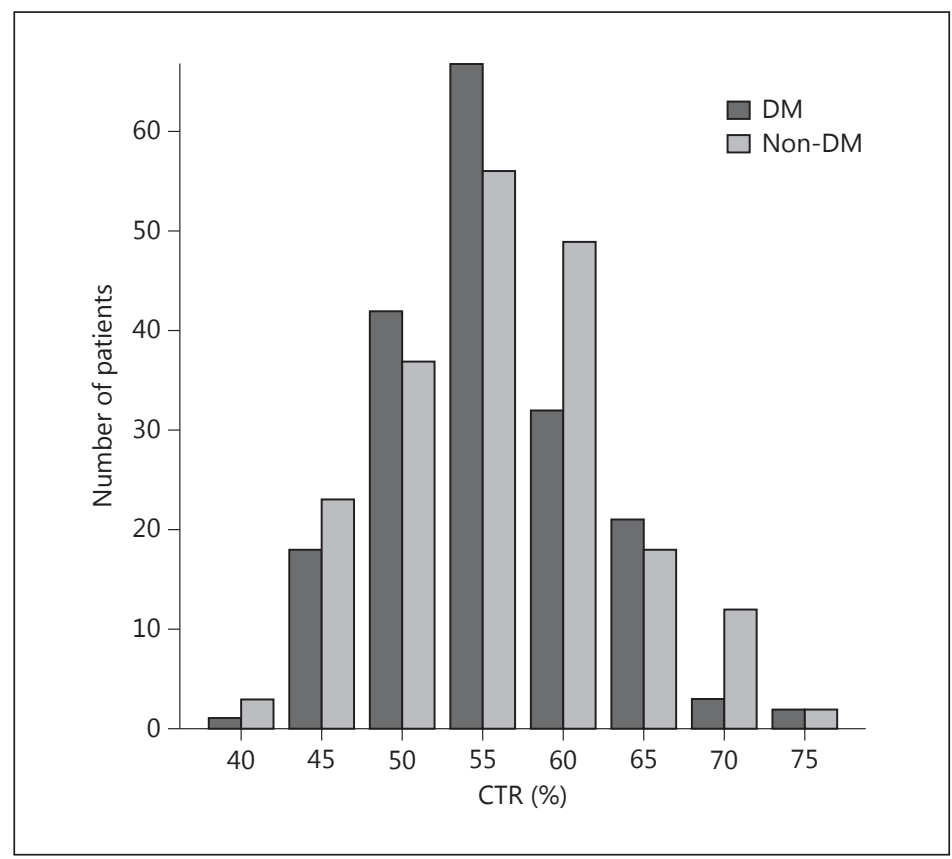

\begin{tabular}{|c|c|c|c|c|}
\hline \multirow[t]{2}{*}{ Versus CTR (\%) } & \multicolumn{2}{|c|}{$\begin{array}{l}\text { Simple linear } \\
\text { regression }\end{array}$} & \multicolumn{2}{|c|}{$\begin{array}{l}\text { Multivariable linear } \\
\text { regression }\end{array}$} \\
\hline & $\mathrm{r}$ & $\mathrm{p}$ & $\begin{array}{l}\text { standardized } \\
\text { coefficient }\end{array}$ & $\mathrm{p}$ \\
\hline Hemoglobin (g/dl) & -0.194 & $<0.001$ & -0.197 & $<0.001$ \\
\hline Age (years) & 0.155 & 0.002 & 0.214 & $<0.001$ \\
\hline BMI & 0.136 & 0.007 & 0.231 & $<0.001$ \\
\hline Albumin (g/dl) & -0.130 & 0.011 & -0.063 & 0.216 \\
\hline
\end{tabular}


Fig. 4. The Kaplan-Meier survival data of all patients grouped according to the CTR (CTR $<50 \%$ vs. $50 \% \leq$ CTR $<55 \%$ vs. CTR $\geq 55 \%$; $\log$-rank $\mathrm{p}=0.002$ ).

Fig. 5. The Kaplan-Meier survival data of patients without IHD grouped according to the CTR (CTR $<50 \%$ vs. $50 \% \leq$ CTR $<55 \%$ vs. CTR $\geq 55 \%$; log-rank p = 0.0298).

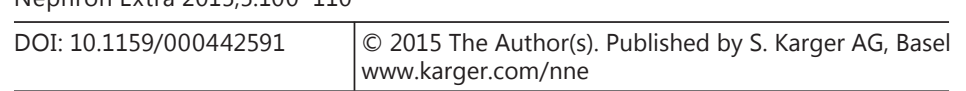

Ito et al.: A Higher Cardiothoracic Ratio Is Associated with 2-Year Mortality after Hemodialysis Initiation
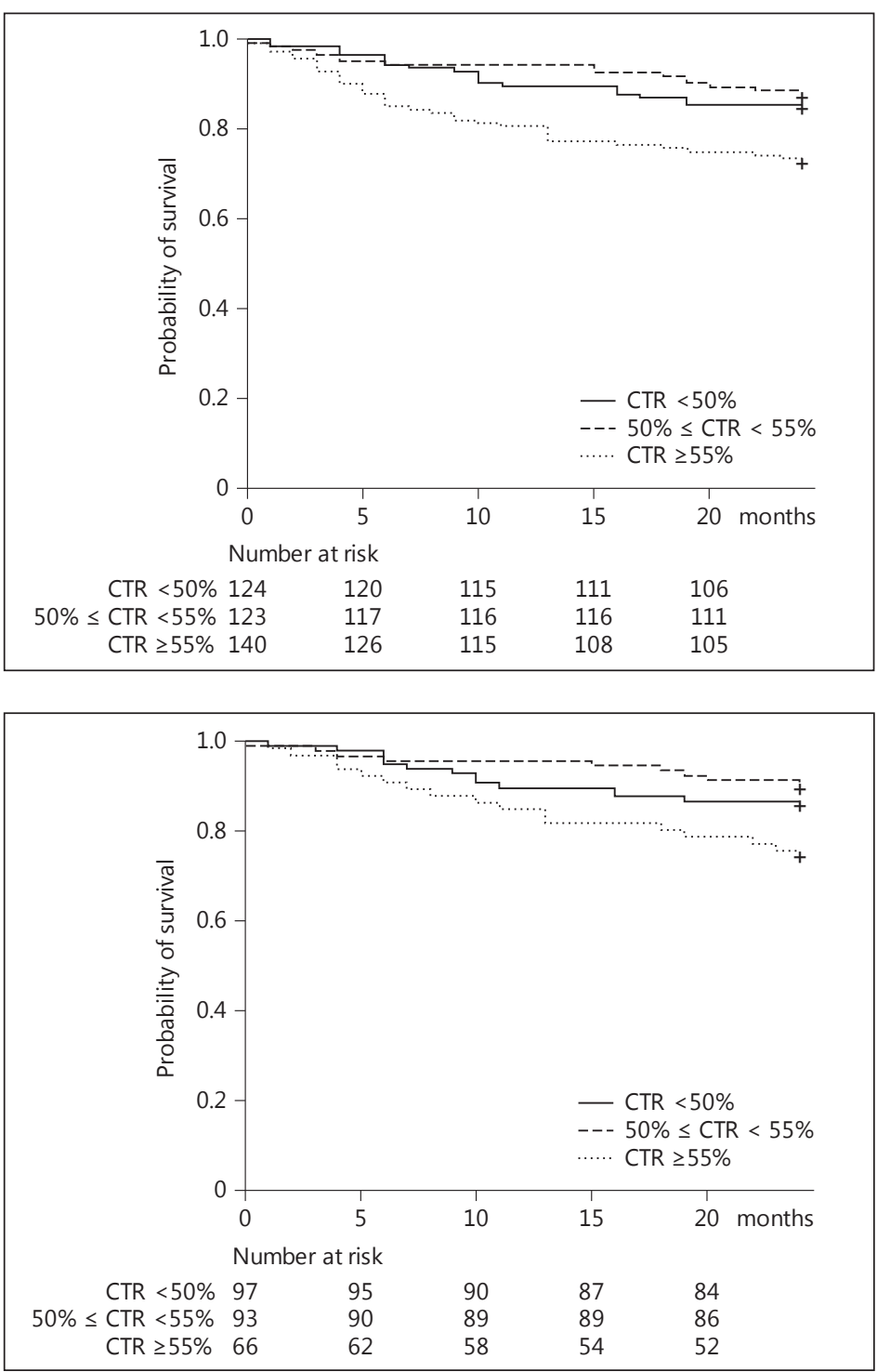

and BMI ( $\mathrm{p}<0.1$ among the three CTR groups), and the presence or absence of DM and eGFR as confounding factors. Age [hazard ratio $(\mathrm{HR})=1.05,95 \%$ confidence interval $(\mathrm{CI})=1.02-$ 1.07], eGFR (HR $=1.14,95 \% \mathrm{CI}=1.06-1.23)$, BMI ( $\mathrm{HR}=0.90,95 \% \mathrm{CI}=0.84-0.96)$, and CTR $\geq 55 \%$ (HR $=1.40,95 \% \mathrm{CI}=1.05-1.88$ ) were found to be independently associated with 2-year all-cause mortality.

\section{Discussion}

The measurement of CTR provides a useful guide for ultrafiltration therapy [13], and a high CTR is reportedly a risk factor for mortality in dialysis patients $[5,6,14]$. The adequate management of body fluids by maintenance dialysis therapy can be performed in these patients. In contrast, in patients with advanced chronic kidney disease (CKD) such as CKD G5, it is sometimes difficult to strictly perform body fluid management even through the use of diuretics because of the decrease in urinary sodium excretion and water retention due to the 
Fig. 6. The Kaplan-Meier survival data of patients with IHD grouped according to the CTR (CTR $<50 \%$ vs. $50 \% \leq \mathrm{CTR}<55 \%$ vs. CTR $\geq 55 \%$; log-rank $\mathrm{p}=0.35$ ).

Fig. 7. The Kaplan-Meier survival data of patients without DM grouped according to the CTR (CTR $<50 \%$ vs. $50 \% \leq$ CTR $<55 \%$ vs. CTR $\geq 55 \%$; log-rank $\mathrm{p}=$ 0.005).

Fig. 8. The Kaplan-Meier survival data of patients with DM grouped according to the CTR (CTR $<50 \%$ vs. $50 \% \leq \mathrm{CTR}<55 \%$ vs. CTR $\geq 55 \%$; log-rank $\mathrm{p}=0.15$ ).

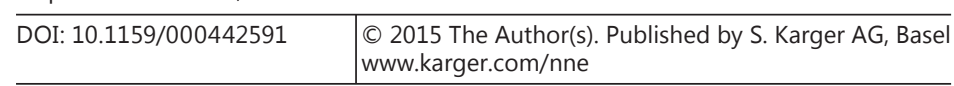

Ito et al.: A Higher Cardiothoracic Ratio Is Associated with 2-Year Mortality after Hemodialysis Initiation
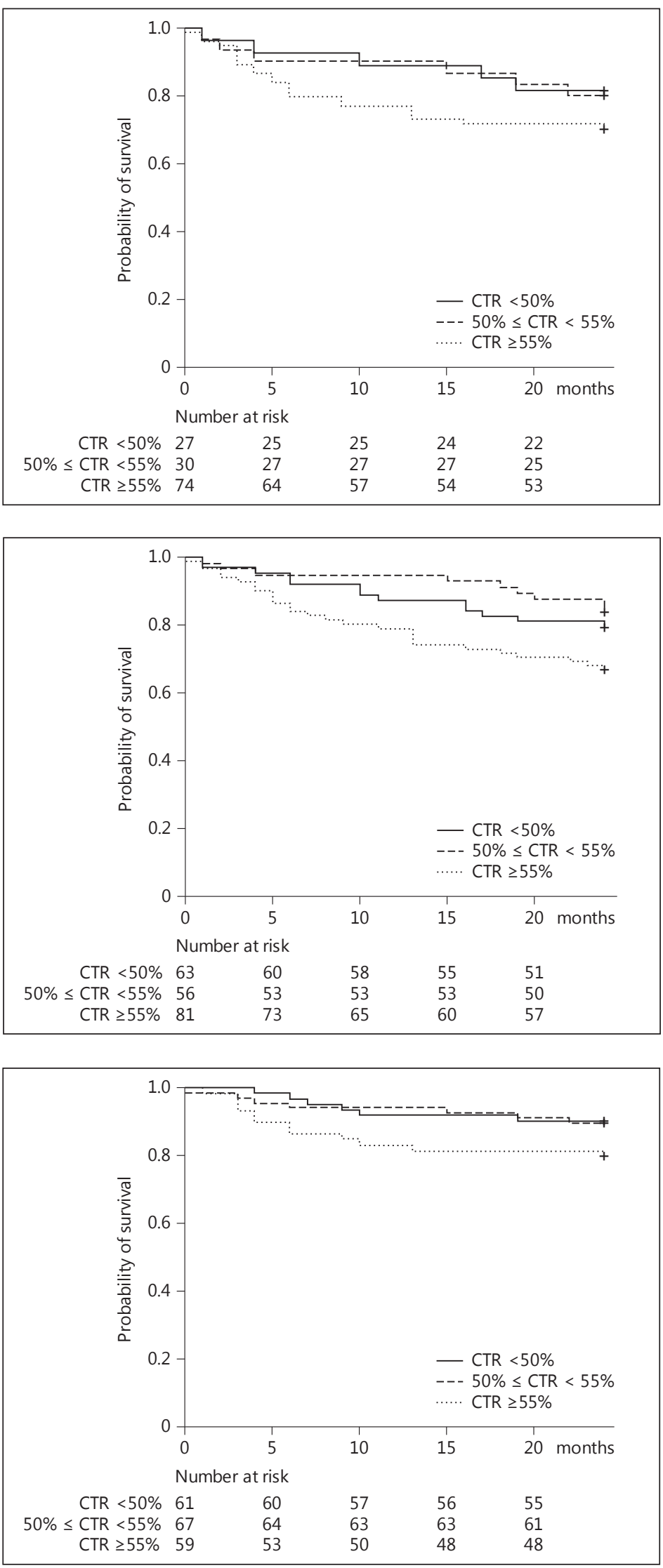
progression of CKD. Thus, patients starting HD may have a higher CTR, because of sodium and water retention, than patients receiving maintenance HD therapy. However, it has been unclear what values of CTR are suitable at the time of HD initiation regarding the prediction of patient mortality after HD initiation. Therefore, this study was performed in three groups of patients categorized by CTR at HD initiation, and our results demonstrated that CTR $\geq 55 \%$ at HD initiation was indicative of poor prognosis in patients undergoing HD during 2 years of observation. Furthermore, CTR differed by the presence or absence of IHD and DM. Patients with cardiomegaly more commonly had a past medical history of IHD than those with normal CTRs [15]. Therefore, our finding that the presence of IHD was associated with a higher CTR is understandable in light of past research. Moreover, the CTR was lower among patients with DM than among those without DM. Generally, DM can be complicated by chronic heart failure and coronary artery disease $[16,17]$; hence, we suspected that the CTR would be higher in patients with DM than in those without DM. However, ESRD patients with DM were more likely to begin dialysis at a higher eGFR [18], and the eGFR among patients with DM also tended to be higher, although the difference was not statistically significant (DM vs. non-DM: $6.0 \pm 2.1$ vs. $5.6 \pm 2.4 \mathrm{ml} / \mathrm{min} / 1.73 \mathrm{~m}^{2}, \mathrm{p}=0.10$ ) in this study. Therefore, the presence of DM might increase the necessity of early HD initiation due to several factors, such as hyperkalemia or the development of uremic symptoms, even in the absence of a cardiac disability including an enlarged CTR.

In this study, we confirmed that there are significant relationships at HD initiation between CTR and hemoglobin levels, age, and BMI in multivariable linear regression analysis, whereas neither C-reactive protein levels, serum albumin concentrations, nor eGFR values show a correlation with these CTR values. It was previously reported that CTR is determined by age, creatinine concentration, corrected calcium concentration, and high-sensitivity $\mathrm{C}$-reactive protein in nondiabetic HD patients, and by age and creatinine concentration in diabetic HD patients [5, 6]. Furthermore, CTR was associated with hemoglobin levels in dialysis patients [19]; therefore, our finding regarding the association between CTR and age and hemoglobin levels at HD initiation is consistent with these previous reports $[5,6,19]$. BMI was also associated with CTR at HD initiation in the current study. For patients receiving HD for more than 6 months, BMI was positively and significantly associated with CTR in a simple linear regression analysis, but the significance disappeared in multiple linear regression analysis [20]. Changes of BMI after HD initiation might influence the difference between our results and those of a previous report [20]. Indeed, BMI decreased after HD initiation, and did not recover [21]. Therefore, BMI at HD initiation may be increased because of the body fluid excess of end-stage CKD patients; however, in this study, the reasons for this association remain unclear and further investigation is necessary.

The association between higher CTR and higher rates of 2-year all-cause mortality found in this study has also been reported in healthy people [22], patients with chronic heart failure [23], and patients undergoing maintenance HD [5, 6]. Furthermore, the analysis based on the values of CTR in patients with IHD or DM did not uncover significant differences in 2-year allcause mortality after HD initiation in this study. Joki et al. [1] reported that the overall prevalence of coronary artery disease was approximately 63\% within 1 month of starting HD, and the presence of IHD or DM could rapidly accelerate cardiac remodeling and/or dysfunction $[24,25]$. Thus, 2-year all-cause mortality in patients with IHD or DM could not be determined using only the CTR at HD initiation because mortality in these patients is believed to be associated with the presence of IHD or DM itself. However, in patients without IHD or DM, an evaluation of the CTR at HD initiation would be useful for predicting a patient's prognosis, and based on these evaluations, further examinations including cardiac ultrasonography should be performed in the clinical setting. Furthermore, this study found that 2-year mortality was independently associated with age, eGFR, BMI, and CTR $>55 \%$. It is well known that older 
age and a high eGFR at HD initiation are associated with mortality [10,11]. Our results also illustrated the importance of these factors in terms of raising mortality risk after HD, as observed in previous reports. Regarding the association between mortality and BMI in patients undergoing HD, a high BMI is likely to be an important factor for survival [26]. In Japan, Aoyagi et al. [27] reported that a low BMI is inversely associated with 2-year mortality, especially in patients undergoing HD who are older than 60 years of age. Furthermore, in patients undergoing maintenance HD, unlike in the general population, mortality risk decreases as BMI increases $[28,29]$. Therefore, our finding of a significant and inverse association between 2-year mortality and BMI is consistent with previous reports [27-29].

This study has several limitations. First, it was a small single-center, retrospective study. Second, the identified independent factors have been consistent predictors of all-cause mortality at HD initiation. Third, an evaluation of treatment and medication after HD initiation could not be performed. Furthermore, whether a high CTR is associated with cardiac mortality remains unclear. Therefore, clarifications of the relationship between the CTR at HD initiation and patients' mortality, especially cardiac mortality, are necessary subjects of future studies.

In conclusion, CTR $>55 \%$ is one of the most important independent factors to predict 2 -year all-cause mortality. Thus, we recommend confirming the cardiac condition of patients at HD initiation with a CTR $>55 \%$ in order to improve their survival.

\section{Acknowledgements}

We thank the study participants and the clinical dialysis center staff, who supported patients undergoing HD and clinical dialysis therapy, in Saitama Prefecture, Japan.

\section{Disclosure Statement}

The authors declare that they have no conflicting interests.

\section{References}

1 Joki N, Hase H, Nakamura R, Yamaguchi T: Onset of coronary artery disease prior to initiation of haemodialysis in patients with end-stage renal disease. Nephrol Dial Transplant 1997;12:718-723.

-2 Nakai S, Hanafusa N, Masakane I, Taniguchi M, Hamano T, Shoji T, Hasegawa T, Itami N, Yamagata K, Shinoda T, Kazama JJ, Watanabe Y, Shigematsu T, Marubayashi S, Morita O, Wada A, Hashimoto S, Suzuki K, Nakamoto H, Kimata N, Wakai K, Fujii N, Ogata S, Tsuchida K, Nishi H, Iseki K, Tsubakihara Y: An overview of regular dialysis treatment in Japan (as of 31 December 2012). Ther Apher Dial 2014;18:535-602.

-3 Stefánsson BV, Brunelli SM, Cabrera C, Rosenbaum D, Anum E, Ramakrishnan K, Jensen DE, Stalhammar NO: Intradialytic hypotension and risk of cardiovascular disease. Clin J Am Soc Nephrol 2014;9:2124-2132.

4 Danzer CS: The cardiothoracic ratio. Am J Med Sci 1919;157:513-521.

5 Chen KH, Lin-Tan DT, Huang WH, Hung CC, Chang CT, Huang JY, Lin JL: Cardiothoracic ratio, malnutrition, inflammation, and two-year mortality in non-diabetic patients on maintenance hemodialysis. Kidney Blood Press Res 2008;31:143-151.

-6 Yen TH, Lin JL, Lin-Tan DT, Hsu KH: Cardiothoracic ratio, inflammation, malnutrition, and mortality in diabetes patients on maintenance hemodialysis. Am J Med Sci 2009;337:421-428.

7 Matsuo S, Imai E, Horio M, Yasuda Y, Tomita K, Nitta K, Yamagata K, Tomino Y, Yokoyama H, Hishida A; Collaborators developing the Japanese equation for estimated GFR: revised equations for estimated GFR from serum creatinine in Japan. Am J Kidney Dis 2009;53:982-992.

-8 Shoji T, Emoto M, Shinohara K, Kakiya R, Tsujimoto Y, Kishimoto H, Ishimura E, Tabata T, Nishizawa Y: Diabetes mellitus, aortic stiffness, and cardiovascular mortality in end-stage renal disease. J Am Soc Nephrol 2001;12: 2117-2124. 
-9 Hayashino Y, Fukuhara S, Akiba T, Akizawa T, Asano Y, Saito A, Bragg-Gresham JL, Ramirez SP, Port FK, Kurokawa K: Diabetes, glycaemic control and mortality risk in patients on haemodialysis: the Japan Dialysis Outcomes and Practice Pattern Study. Diabetologia 2007;50:1170-1177.

10 Pan Y, Xu XD, Guo LL, Cai LL, Jin HM: Association of early versus late initiation of dialysis with mortality: systematic review and meta-analysis. Nephron Clin Pract 2012;120:c121-c131.

11 Rosansky SJ, Eggers P, Jackson K, Glassock R, Clark WF: Early start of hemodialysis may be harmful. Arch Intern Med 2011;171:396-403.

12 Kanda Y: Investigation of the freely available easy-to-use software 'EZR' for medical statistics. Bone Marrow Transplant 2013;48:452-458.

13 Poggi A, Maggiore Q: Cardiothoracic ratio as a guide to ultrafiltration therapy in dialyzed patients. Int J Artif Organs 1980;3:332-337.

14 Ozkahya M, Ok E, Toz H, Asci G, Duman S, Basci A, Kose T, Dorhout Mees EJ: Long-term survival rates in haemodialysis patients treated with strict volume control. Nephrol Dial Transplant 2006;21:3506-3513.

15 Baker NC, Assal C, Shirani J: Clinical significance of cardiomegaly caused by cardiac adiposity. Am J Cardiol 2012;109:1374-1378.

16 Stone PH, Muller JE, Hartwell T, York BJ, Rutherford JD, Parker CB, Turi ZG, Strauss HW, Willerson JT, Robertson T, Braunwald E, Jaffe AS; MILIS Study Group: The effect of diabetes mellitus on prognosis and serial left ventricular function after acute myocardial infarction: contribution of both coronary disease and diastolic left ventricular dysfunction to the adverse prognosis. The MILIS Study Group. J Am Coll Cardiol 1989;14:49-57.

17 Kannel WB: Framingham study insights on diabetes and cardiovascular disease. Clin Chem 2011;57:338-339. Lassalle M, Labeeuw M, Frimat L, Villar E, Joyeux V, Couchoud C, Stengel B: Age and comorbidity may explain the paradoxical association of an early dialysis start with poor survival. Kidney Int 2010;77:700-707.

19 Asakawa T, Joki N, Tanaka Y, Hayashi T, Hase H, Komatsu Y, Ando R, Ikeda M, Inaguma D, Sakaguchi T, Shinoda T, Koiwa F, Negi S, Yamaka T, Shigematsu T: Association between the hemoglobin level and cardiothoracic ratio in patients on incident dialysis. Cardiorenal Med 2014;4:189-200.

20 Yen TH, Lin JL, Lin-Tan DT, Hsu CW: Association between body mass and mortality in maintenance hemodialysis patients. Ther Apher Dial 2010;14:400-408.

-21 Badve SV, Paul SK, Klein K, Clayton PA, Hawley CM, Brown FG, Boudville N, Polkinghorne KR, McDonald SP, Johnson DW: The association between body mass index and mortality in incident dialysis patients. PLoS One 2014;9:e114897.

-22 Hemingway H, Shipley M, Christie D, Marmot M: Cardiothoracic ratio and relative heart volume as predictors of coronary heart disease mortality. The Whitehall study 25 year follow-up. Eur Heart J 1998;19:859-869.

-23 Giamouzis G, Sui X, Love TE, Butler J, Young JB, Ahmed A: A propensity-matched study of the association of cardiothoracic ratio with morbidity and mortality in chronic heart failure. Am J Cardiol 2008;101:343-347.

-24 Pfeffer MA, Braunwald E: Ventricular remodeling after myocardial infarction. Experimental observations and clinical implications. Circulation 1990;81:1161-1172.

25 Boudina S, Abel ED: Diabetic cardiomyopathy revisited. Circulation 2007;115:3213-3223.

-26 Abbott KC, Glanton CW, Trespalacios FC, Oliver DK, Oritz MI, Aqodoa LY, Cruess DF, Kimmel PL: Body mass index, dialysis modality, and survival: analysis of the United States Renal Data System Dialysis Morbidity and Mortality Wave II Study. Kidney Int 2004;65:597-605.

-27 Aoyagi T, Naka H, Miyaji K, Hayakawa K, Ishikawa H, Hata M: Body mass index for chronic hemodialysis patients: stable hemodialysis and mortality. Int J Urol 2001;8:S71-S75.

28 Leavey SF, McCullough K, Hecking E, Goodkin D, Port FK, Young EW: Body mass index and mortality in 'healthier' as compared with 'sicker' haemodialysis patients: results from the Dialysis Outcomes and Practice Patterns Study (DOPPS). Nephrol Dial Transplant 2001;16:2386-2394.

-29 Kalantar-Zadeh K, Block G, Humphreys MH, Kopple JD: Reverse epidemiology of cardiovascular risk factors in maintenance dialysis patients. Kidney Int 2003;63:793-808. 